Original Article

Orthopedics and Traumatology
Medical Journal of Islamic World Academy of Sciences

doi: 10.5505/ias.2019.25901 2019;27(1): 1-4

\title{
Role of arthroscopic surgery in patients aged 60 years and more with degenerative knee
}

\author{
Abuzer ULUDAĞ \\ Department of Orthopedics and Traumatology, Faculty of Medicine, Adiyaman University, Adiyaman, Turkey \\ Correspondence \\ Abuzer ULUDAĞ \\ Adıyaman Üniversitesi Tıp Fakültesi Ortopedi ve Travmatoloji Kliniği 02100 Adıyaman, Türkiye \\ e-mail: uludagabuzer@gmail.com
}

\section{ABSTRACT}

Osteoarthritis is a progressive degenerative disease of the synovial joints that causes pain and limitation of range of motion. Surgery is an effective treatment method for this when conservative treatment is ineffective. Arthroscopy is one of the surgical methods that is controversial for degenerative knees. This study aimed to find the efficacy of arthroscopy in patients aged 60 years and more with degenerative knees.

A total of 27 patients (16 women and 11 men), aged 60 years and more, operated between 2012 and 2015, in whom conservative treatment for 3 months was ineffective before surgery, were enrolled. These patients had osteoarthritis according to the American College of Rheumatology criteria and a Kellgren-Lawrence Osteoarthritis Index score of less than 4. The Visual Analog Scale (VAS) and Western Ontario and McMaster Universities (WOMAC) Osteoarthritis Index of patients before and after arthroscopy were compared by age, gender, and complications.

The average Kellgren-Lawrence Index was 2.4. The average VAS score was 8 before surgery and 4.1 after surgery, indicating an almost 50\% improvement in pain. The average WOMAC Osteoarthritis Index was $40.7 \%$ before surgery and $25.5 \%$ after surgery. No complications were observed after surgery. The preoperative and postoperative VAS and WOMAC values were significant. Both the described pain and osteoarthritis of the patients were partially recovered after arthroscopic surgery.

In conclusion, arthroscopy treated functions that increased pain and mechanical symptoms and hence was beneficial in appropriately selected degenerative knees.

Key words: Arthroscopy, debridement, degenerative knee, knee pathologies, lavage

\section{INTRODUCTION}

Osteoarthritis of the synovial joints is a progressive degenerative disease, which especially affects the cartilage and also bone, tendon, synovium, and meniscus. Osteoarthritis generally affects the knee, hip, and wrist, and can interrupt the daily activities of patients by causing pain, joint stiffness, and motion disabilities. The main purpose of treating osteoarthritis is to recover the functions of joints by reducing pain and joint stiffness. Patients generally opt for surgery when conservative treatment fails to improve their condition. One of the surgical methods for treating osteoarthritis is arthroscopy. Arthroscopy includes washing joints, debridement of tear and fringing cartilage, removing free fragments in joints, debridement of meniscus, repairing meniscus tear, resection of synovial hypertrophy, and subchondral drilling. However, this surgery has been controversial. This study aimed to investigate the efficacy of arthroscopy in patients aged 60 years and more with degenerative knees in whom conservative treatment was ineffective $(1,2)$.

\section{MATERIALS AND METHODS}

A total of 39 patients, aged 60 years and more, operated between 2012 and 2015, in whom conservative treatment for 3 months was ineffective before surgery, were examined retrospectively. Patients with osteoarthritis according to the American College of Rheumatology criteria and a Kellgren-Lawrence Osteoarthritis Index score less than 4 were included in this study. Therefore, a total of 27 patients were enrolled for the study. 
All patients underwent spinal anesthesia and used tourniquet before surgery. Anteromedial and anterolateral portals were used in all patients. No extra portals were needed. The patients who underwent meniscectomy and debridement were allowed to step on the first day. The other patients who had meniscus fixing, femoral drilling-microfracture, and mosaicplasty were allowed to step progressively after 4th week. The age, gender, and mobilization of the patients were compared according to the Visual Analog Scale (VAS) and Western Ontario and McMaster Universities (WOMAC) Osteoarthritis Index.

The data were evaluated using paired-samples $t$ test. The difference in genders was compared using the Student $t$ test. A $P$ value $<0.05$ was considered statistically significant.

\section{RESULTS}

A total of 27 patients (16 women and 11 men) were analyzed retrospectively. Average age was 65.2 years for women and 62.9 years for men. Surgery was performed on 18 left knees and 9 left knees. The average tracking time was 31 months. According to the Kellgren-Lawrence Index, 17 patients had grade 2 osteoarthritis and 10 had grade 3 osteoarthritis. The average Kellgren-Lawrence index was 2.4. Except one patient, the others had unrepairable degenerative meniscus tear. In total, 18 patients were treated by meniscectomy and lavage; 1 patient by lavage and debridement (abrasion-plasty); 5 patients by meniscectomy and femoral drilling-microfracture; and 1 patient by meniscectomy and mosaicplasty. The average VAS score was 8 before surgery and 4.1 after surgery, indicating almost a $50 \%$ decrease in pain. The average WOMAC Osteoarthritis Index was $40.7 \%$ before surgery and $25.5 \%$ after surgery. No complications were found after the surgery. The preoperative and postoperative VAS and WOMAC values were considered significant $(P<0.001)$. With these values, both the described pain and osteoarthritis of the patients were partially recovered after arthroscopic surgery. Statistical analysis of postoperative pain described by gender was significant $(P=0.039)$; other values were not significant. These findings showed that women still had more pain than men after surgery (Table 1).

\section{DISCUSSION}

Osteoarthritis is a progressive degenerative disease of the synovial joints in patients aged 40 years and more. Knee is the most affected joint in osteoarthritis. The common complaint is pain, and most patients need surgical treatment. Arthroscopic debridement, implantation of cartilage, correction of axle (tibial/femoral osteotomies), and arthroplasty are the primary surgical choices (4).

Arthroscopic treatment in degenerative knees has many advantages. The intra-articular rashes of cartilage seen in osteoarthritis increase the concentration of tumor necrosis factor alpha (TNF-a). TNF- $a$ causes cartilage destruction and joint pain. Also, surplus calcium crystals spark inflammation. Moreover, it can cause progression of osteoarthritis and symptoms by increasing cartilage destruction (6). By removing these components from joints by arthroscopic debridement and lavage can slow down the inflammation process. However, which patients need arthroscopy and when is controversial. Differences in patient populations, different protocols, subjective patient statements, and no standardized criteria have led to this controversy. Uncontrolled studies suggest that arthroscopic surgery is beneficial in osteoarthritis. However, wide controlled studies suggest that arthroscopic surgery should not be the primary treatment choice and can be beneficial in just few chosen patients (1,7-9).

Stefan et al. showed that arthroscopic irrigation decreased $80 \%$ of complaints of patients aged 60 years and more. However, meniscectomy increased $230 \%$ by that time. According to their study, arthroscopic lavage was not much beneficial for degenerative and mechanical symptoms in knees. In this study, arthroscopic lavage was performed in just one patient (3.5\%). The other patients underwent other arthroscopic treatments.

Many surgeons advocate that arthroscopy is helpful in patients with less degenerative knees $(7,10)$. Shannon et al. followed up 54 patients, with an average age of 60.9 years, who had less- and medium-degree osteoarthritis. They found that 19 patients required meniscectomy and loose body excision and others required diagnostic arthroscopy and lavage. Moreover, 37 patients (68.5) were cured subjectively. 
Table 1: Laboratory values of patients at various stages of two-stage revision surgery.

\begin{tabular}{|c|c|c|c|c|c|c|c|c|c|c|}
\hline \multirow[t]{2}{*}{ Patient } & \multirow[t]{2}{*}{$\begin{array}{l}\text { Gender } \\
\text { (male- } \\
\text { female) }\end{array}$} & \multirow[t]{2}{*}{$\begin{array}{l}\text { Age } \\
\text { (year) }\end{array}$} & \multirow[t]{2}{*}{$\begin{array}{l}\text { Side } \\
\text { (right- } \\
\text { left) }\end{array}$} & \multirow[t]{2}{*}{$\begin{array}{l}\text { Follow-up } \\
\text { (months) }\end{array}$} & \multirow[t]{2}{*}{$\begin{array}{l}\text { Kellgren- } \\
\text { Lawrence } \\
\text { index }\end{array}$} & \multirow[t]{2}{*}{ Applied surgery } & \multicolumn{2}{|c|}{$\begin{array}{c}\text { WOMAC } \\
\text { Osteoarthritis } \\
\text { Index Score } \\
\mathrm{p}<0.001\end{array}$} & \multicolumn{2}{|c|}{$\begin{array}{l}\text { VAS Score } \\
p<0.001\end{array}$} \\
\hline & & & & & & & $\begin{array}{l}\text { Preop } \\
(\%)\end{array}$ & $\begin{array}{l}\text { Postop } \\
(\%)\end{array}$ & Preop & Postop \\
\hline 1 & $\mathrm{~F}$ & 68 & $\mathrm{~L}$ & 78 & 3 & Menisectomy-drilling & 21,80 & 37,50 & 8 & 4 \\
\hline 2 & M & 65 & $\mathrm{R}$ & 81 & 2 & Menisectomy & 72,90 & 12,0 & 8 & 2 \\
\hline 3 & $\mathrm{~F}$ & 72 & $\mathrm{~L}$ & 81 & 3 & Meniscectomy-drilling & 70,80 & 54,00 & 9 & 4 \\
\hline 4 & $\mathrm{~F}$ & 69 & $\mathrm{R}$ & 72 & 2 & Meniscectomy-drilling & 17,70 & 9,30 & 9 & 2 \\
\hline 5 & $\mathrm{~F}$ & 67 & $L$ & 38 & 3 & Menisectomy & 32,20 & 17,70 & 9 & 2 \\
\hline 6 & M & 62 & $\mathrm{~L}$ & 24 & 2 & Menisectomy & 27,00 & 15,60 & 7 & 2 \\
\hline 7 & M & 67 & $\mathrm{~L}$ & 24 & 2 & Menisectomy & 9,30 & 2,10 & 4 & 1 \\
\hline 8 & M & 62 & $L$ & 21 & 2 & Menisectomy & 39,50 & 19,80 & 8 & 2 \\
\hline 9 & $\mathrm{~F}$ & 62 & $\mathrm{~L}$ & 20 & 2 & Menisectomy-microfracture & 44,70 & 38,50 & 8 & 8 \\
\hline 10 & $\mathrm{~F}$ & 72 & $L$ & 16 & 3 & Menisectomy-drilling & 46,80 & 29,10 & 8 & 4 \\
\hline 11 & $\mathrm{~F}$ & 65 & $\mathrm{~L}$ & 16 & 3 & Menisectomy & 31,20 & 21,80 & 8 & 5 \\
\hline 12 & $\mathrm{~F}$ & 65 & $\mathrm{R}$ & 16 & 2 & Menisectomy & 60,40 & 30,10 & 9 & 5 \\
\hline 13 & $\mathrm{~F}$ & 68 & $L$ & 13 & 3 & Menisectomy-debridement & 32,20 & 4,10 & 9 & 2 \\
\hline 14 & $\mathrm{~F}$ & 66 & $\mathrm{~L}$ & 13 & 3 & Menisectomy & 47,90 & 28,10 & 8 & 6 \\
\hline 15 & $\mathrm{~F}$ & 65 & $\mathrm{~L}$ & 12 & 3 & Menisectomy-debridement & 51,00 & 31,20 & 7 & 4 \\
\hline 16 & M & 61 & $\mathrm{R}$ & 12 & 2 & Menisectomy & 20,80 & 5,20 & 9 & 3 \\
\hline 17 & M & 67 & $\mathrm{R}$ & 11 & 2 & Menisectomy-debridement & 50,00 & 13,54 & 8 & 2 \\
\hline 18 & $\mathrm{~F}$ & 69 & $L$ & 11 & 3 & Menisectomy-debridement & 52,00 & 33,33 & 8 & 6 \\
\hline 19 & M & 61 & $L$ & 10 & 2 & Menisectomy-debridement & 37,50 & 27,08 & 9 & 5 \\
\hline 20 & M & 64 & $L$ & 37 & 2 & Menisectomy & 27,00 & 46,87 & 7 & 6 \\
\hline 21 & $\mathrm{~F}$ & 64 & $\mathrm{R}$ & 38 & 2 & Menisectomy & 79,10 & 70,04 & 8 & 8 \\
\hline 22 & $\mathrm{~F}$ & 66 & $L$ & 36 & 3 & Menisectomy & 24,70 & 16,00 & 9 & 4 \\
\hline 23 & M & 73 & $L$ & 38 & 2 & Menisectomy-debridement & 45,80 & 15,60 & 9 & 5 \\
\hline 24 & $\mathrm{~F}$ & 66 & $\mathrm{R}$ & 24 & 3 & Menisectomy & 28,10 & 28,10 & 8 & 7 \\
\hline 25 & M & 68 & $\mathrm{R}$ & 24 & 2 & Menisectomy-mosaicplasty & 32,20 & 25,00 & 6 & 5 \\
\hline 26 & M & 68 & $L$ & 56 & 2 & Menisectomy & 42,70 & 21,80 & 8 & 2 \\
\hline 27 & $\mathrm{~F}$ & 68 & $\mathrm{R}$ & 56 & 2 & Menisectomy & 37,50 & 26,00 & 8 & 5 \\
\hline
\end{tabular}

This indicated that arthroscopy was helpful in chosen patients. Law et al. (10) treated 180 knees of 169 patients with arthroscopy who had an average Kellgren-Lawrence Index of 2.02. Arthroscopic treatment satisfied the expectations of $96 \%$ patients. Specified arthroscopic surgery was helpful for patients who had symptomatic patellofemoral arthritis, degenerative meniscus tear, and symptomatic chondral flap. In the present study, $92 \%$ of the patients were satisfied with arthroscopic surgery. The pain scores decreased in $50 \%$ of patients. Functional activities eased in $62 \%$ of patients. Therefore, surgery was considered successful in patients with Kellgren-Lawrence Osteoarthritis Index of 2.2, and $96 \%$ of patients had mechanical knee problems.

Sannon et al. (4) suggested that arthroscopic surgery could be considered as a primary surgical option for osteoarthritis. Thorlund et al. (11) not- 
ed a reduction in arthroscopic surgery in the last 2 years. Dhawan et al. (12) examined preoperative arthroplasty on 12,806 degenerative knees in the last 4 years and found that 19\% of these patients had arthroscopic surgery before arthroplasty. However, $96 \%$ of the patients who had arthroscopic surgery had arthroplasty within 3 years. The patients in this study were followed up for an average of 31 months. Followed-up patients under 3 years was $77 \%$ mainly high. Arthroscopy was beneficial in short follow-up. However, more follow-up time is needed to assess the need for arthroscopy.

The complications of arthroscopy were very few
(2-4). In this study, no complications were encountered during intraoperative and postoperative periods or follow-ups.

In conclusion, arthroscopy was beneficial before arthroplasty in patients with grade 2-3 osteoarthritis, and especially in those with degenerative mechanical complaints that cannot be cured with conservative treatment. Moreover, arthroscopy had few complications.

\section{ACKNOWLEDGMENT}

The author would like to thank for data collection to Assoc. Prof. Dr. H. Bayram TOSUN.

\section{REFERENCES}

1. Karaali E, Duramaz A,Öneş HN, Bilgili MG, Erçin E, Avcan MCl. Early and mid term results and life quality of the patients who underwent to arthroscopic cartilage debridement and joint lavage because of symptomatic gonarthrosis. Medical Journal of Bakirköy 2014; 10: 116-120.

2. Lazic S, Boughton O, Hing C, Bernard, J Arthroscopic washout of the knee: a procedure in decline.The Knee2014; 21(2): 631634.

3. Kellgren JH, Lawrence JS. Radiological assessment of osteoarthrosis. Annals of the rheumatic diseases 1957; 16.4: 494-490

4. Shannon F. Devitt A. Poynton A. Fitzpatrick P.Walsh M. Shortterm benefit of arthroscopic washout in degenerative arthritis of the knee. International orthopaedics 2001; 25: 242-245.

5. Cameron DM. Holland C. Hungerford DS. Frondoza, CG Cartilage debris increases the expression of chondrodestructive tumor necrosis factor-a by articular chondrocytes. The Journal of Arthroscopic \& Related Surgery 2004 ; 20:1040-1043.

6. Rosenthal AK. Crystals, inflammation, and osteoarthritis. Current opinion in rheumatology 2011; 23: 170-180.

7. Lee DJ. John CE. Utility of arthroscopic surgery for osteoarthritis of the knee. Geriatric orthopaedic surgery and rehabilitation $2015 ; 6: 47-49$.

8. Moseley JB. O'malley K. Petersen NJ. Menke TJ. Brody BA. Kuykendall DH. Wray NP. A controlled trial of arthroscopic surgery for osteoarthritis of the knee. New England Journal of Medicine 2002; 347:81-88

9. Arthroscopy Association of Canada, Wong I. Hiemstra L. Ayeni OR. Getgood A. Beavis C. Litchfield R. Position Statement of the Arthroscopy Association of Canada (AAC) Concerning Arthroscopy of the Knee Joint-September 2017." Orthopaedic journal of sports medicine 2018; 6: 596-597.

10. Law GW. Lee JK. Soong J. Lim JWS. Zhang KT. Tan AHC. Arthroscopic debridement of the degenerative knee-ls there still a role?. Asia-Pacific journal of sports medicine, arthroscopy, rehabilitation and technology 2019; 15: 23-28.

11. Thorlund JB. Juhl CB. Roos EM. Lohmander LS. Arthroscopic surgery for degenerative knee: systematic review and metaanalysis of benefits and harms. bmj 2015; 350: h2747.

12. Dhawan A. Mather RC. Karas V. Ellman MB. Young BB. Bach JBR. Cole BJ. An epidemiologic analysis of clinical practice guidelines for non-arthroplasty treatment of osteoarthritis of the knee. Arthroscopy: The Journal of Arthroscopic \& Related Surgery $2014 ; 30: 65-71$. 